\title{
Volatile oils of Chinese crude medicines exhibit antiparasitic activity against human Demodex with no adverse effects in vivo
}

\author{
JI-XIN LIU ${ }^{1}$, YAN-HONG SUN ${ }^{1}$ and CHAO-PIN LI ${ }^{2}$ \\ ${ }^{1}$ Department of Clinical Pathogen Biology, Qiqihar Medical University, Qiqihar, Heilongjiang 161006; \\ ${ }^{2}$ Department of Medical Parasitology, Wannan Medical College, Wuhu, Anhui 241002, P.R. China
}

Received May 14, 2014; Accepted December 18, 2014

DOI: $10.3892 /$ etm.2015.2272

\begin{abstract}
Demodex is a type of permanent obligatory parasite, which can be found on the human body surface. Currently, drugs targeting Demodex usually result in adverse effects and have a poor therapeutic effect. Thus, the aim of the present study was to investigate the use of Chinese crude medicine volatile oils for targeting and inhibiting Demodex in vitro. The volatile oils of six Chinese crude medicines were investigated, including clove, orange fruit, Manchurian wildginger, cinnamon bark, Rhizome Alpiniae Officinarum and pricklyash peel, which were extracted using a distillation method. The exercise status of Demodex folliculorum and Demodex brevis and the antiparasitic effects of the volatile oils against the two species were observed using microscopy. A skin irritation test was used to examine the irritation intensity of the volatile oils. In addition, an acute toxicity test was utilized to observe the toxicity effects of the volatile oils on the skin. Xin Fumanling ointment was employed as a positive control to identify the therapeutic effects of the volatile oils. The results indicated that all six volatile oils were able to kill Demodex efficiently. In particular, the clove volatile oil was effective in inducing optimized anti-Demodex activity. The lethal times of the volatile oils were significantly decreased compared with the Xin Fumanling ointment $(\mathrm{P}<0.05)$. Furthermore, the skin irritation test results indicated that the clove volatile oil did not trigger any irritation ( 0.2 and 0.3 points for intact and scratched skin, respectively), and had a safety equal to that of distilled water. There were not any adverse effects observed following application of the clove volatile oil on the intact or scratched skin. In conclusion, the volatile oils of Chinese crude medicines, particularly that of clove, demonstrated an evident anti-Demodex activity and were able to kill Demodex effectively and safely in vivo.
\end{abstract}

Correspondence to: Dr Ji-Xin Liu, Department of Clinical Pathogen Biology, Qiqihar Medical University, 333 Bukui Street, Qiqihar, Heilongjiang 161006, P.R. China

E-mail: 1jx_2000_1979@163.com

Key words: Chinese crude medicine, volatile oil, Demodex, anti-Demodex activity

\section{Introduction}

Demodex, a type of permanent obligatory parasite (class, Arachnida; superorder, Acariformes), is an elongated ectoparasite found on the human body surface, including the face, cheeks, forehead, nose and eyelids (1). There are numerous species of Demodex, but only Demodex folliculorum and Demodex brevis are found on the human body $(2,3)$. The organism, Demodex folliculorum, is found in the eyelash follicle, while Demodex brevis burrows deep into the sebaceous and meibomian glands (3). The rate of Demodex infestation increases with age, with the organism identified in $84 \%$ of the population at age 60 and in $100 \%$ of those aged $>70$ years (4). In addition to the higher density of Demodex observed in patients with rosacea (5), Demodex has also been suggested as a cause of other skin diseases, including pityriasis folliculorum, perioral dermatitis $(6,7)$, scabies-like eruptions, facial pigmentation, eruptions of the bald scalp, demodicosis gravis and even basal cell carcinoma $(8,9)$. Although the pathogenic role remains elusive, efforts have been made to eradicate ocular Demodex in patients presenting with blepharitis (10).

To date, a number of studies $(6,7,10)$ have investigated various drugs targeting Demodex; however, the majority of these drugs were not found to be effective. There are seldom studies investigating the antiparasitic effects of volatile oils from Chinese crude drugs against Demodex. Thus, the present study selected six types of Chinese crude drugs, among the 300 drugs that are rich in volatile oil. Subsequently, the anti-Demodex efficacy and safety were studied for the six volatile oils used in the Demodex therapy.

\section{Materials and methods}

Materials, reagents and animals. Clove, orange fruit, Manchurian wild ginger, cinnamon bark, Rhizoma Alpiniae Officinarum and pricklyash peel were purchased from Huainan Hengkang Medicine Co., Ltd. (Huainan, China). The quality of these drugs was assessed by Professor Genbao Zhang in the Department of Pharmacy (Qiqihar Medical University, Qiqihar, China). An optical microscope and photographic system were purchased from Nikon Corporation (model SMZ1500, Tokyo, Japan). The clinical anti-Demodex drug, Xin Fumanling ointment, was purchased from Shandong Jiankang Pharmaceutical Co., Ltd. (Jinan, China). In total, four male rabbits and four 
A

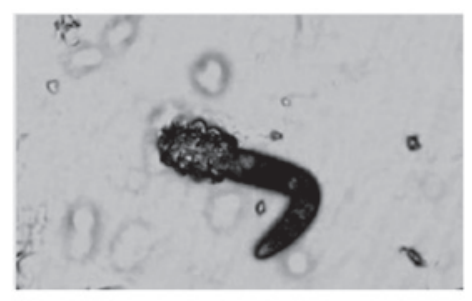

C

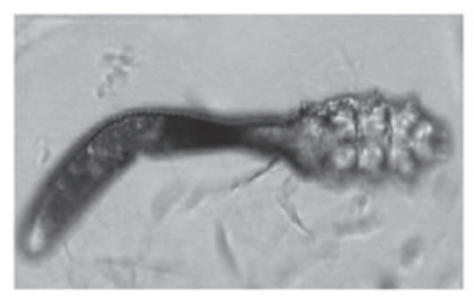

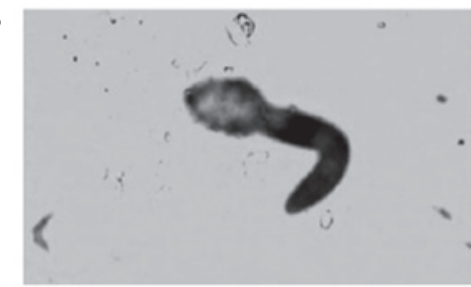

D

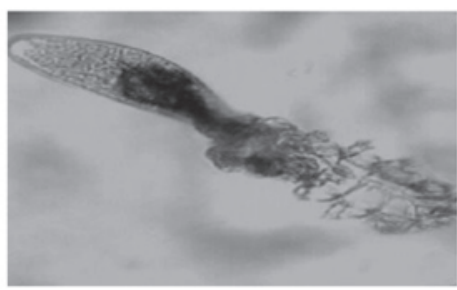

Figure 1. Mechanism underlying the antiparasitic process of the clove volatile oil on Demodex folliculorum. (A) Prior to administration of the volatile oil; (B) following full contact of Demodex with the volatile oil; (C) Demodex folliculorum appears marcid; (D) transparent body of Demodex folliculorum.

female rabbits (weight, $2.0-2.5 \mathrm{~kg}$ ) were provided by the Experimental Animal Base of Anhui University of Science and Technology (Huainan, China). The eight rabbits were divided into two groups randomly; one group received treatment with the volatile oils, while the other group received Xin Fumanling ointment treatment. Furthermore, sodium chloride solution was selected as the negative control. The present study was approved by the Ethics Committee of Qiqihar Medical University.

Extraction of volatile oil. In total, 50-g samples of each Chinese crude medicine were smashed, filtered and soaked for $8 \mathrm{~h}$. The extraction of volatile oil was performed using a distillation method, followed by drying with sodium sulfate. The detailed procedures were performed according to previous studies $(11,12)$.

In vitro experiment. Since there is a high prevalence of Demodex in patients with cylindrical dandruff, lashes with cylindrical dandruff were selected for the in vitro experiment (13). A total of 20 patients were selected to obtain the Demodex, these patients were diagnosed by the Department of Dermatology (Qiqihar Medical University). Informed consent was obtained from all of the patients. Demodex was obtained with cellulose tape at room temperature $\left(30 \pm 0.5^{\circ} \mathrm{C}\right)$. The experiment was performed in a good manufacturing practice laboratory. Volatile oil was dripped onto the Demodex with a micropipette, and the movements of the Demodex body and legs were observed continuously under a microscope (CX31; Olympus, Tokyo, Japan) at x40 magnification for $60 \mathrm{~min}$. Death of the parasite was defined as no movements of the body and no changes to the legs for a minimum of $30 \mathrm{sec}$. The lethal time was defined as the time between the addition of the solution and when the organism stopped moving. Xin Fumanling ointment was used as the positive control, while saline served as the blank control.

Skin irritation test. In total, a $0.3-\mu 1$ sample of each volatile oil was applied to a section of the rabbit skin with the size of $2.5 \times 2.5 \mathrm{~cm}$. The skin was fixed with the bandage. Exposure time to the volatile oil lasted for $24 \mathrm{~h}$, after which fresh saline was applied to the same site for clearing of the retained volatile oils.

Measurements of skin irritation. Assessments of skin irritation were performed according to two criteria (14). The first criteria was scored as follows: No erythema, 0 points; speckling, moderate erythema, 1 point; uniform, moderate erythema, 2 points; intense erythema, 3 points; and intense erythema (red hot) with edema, 4 points. The second criteria was scored as follows: No edema, 0 points, extremely slight edema, 1 point; slight edema, 2 points; moderate edema, 3 points; and serious edema, 4 points. The skin irritation score was evaluated by combining the two criteria. No irritation was defined as 0.0-0.4 points, slight irritation was classified as $0.5-1.9$ points, moderate irritation was defined as 2.0-5.9 points and intense irritation was classified as 6.0-8.0 points.

Acute toxicity test. Acute toxicity of the volatile oils was investigated using the rabbits treated with Demodex. Observations of the rabbits were conducted for one week continuously. The aim of this experiment was to observe the systemic toxicity and mortality of the rabbits, including changes to the skin, hair, eyes and mucosa, changes to the respiratory system, circulatory system and central nervous system, limbs activities and behavioral style. In particular, observations of a tremble and muzziness were recorded. The criteria used for assessing acute toxicity was performed according to previous studies $(15,16)$.

Statistical analysis. Data are expressed as the mean \pm standard deviation, and were analyzed using Microsoft Excel (Microsoft, Redmont, WA, USA). The data between groups were evaluated using a two-tailed t-test, where $\mathrm{P}<0.05$ was considered to indicate a statistically significant difference.

\section{Results}

Antiparasitic activity of the volatile oils. Sodium chloride was used as a negative control and was shown to have no effects on the rabbits (data not shown). Prior to the addition of the 
A

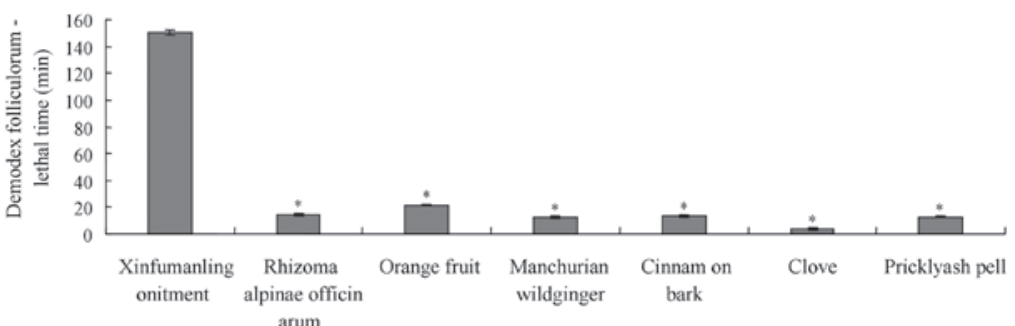

B

Volatile oil

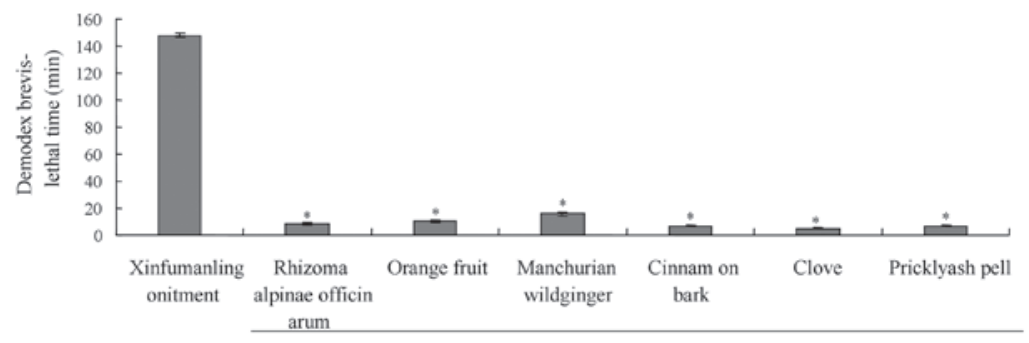

Volatile oil

Figure 2. Lethal times of the six volatile oils on (A) Demodex folliculorum and (B) Demodex brevis. "P<0.05, vs. Xin Fumanling ointment.

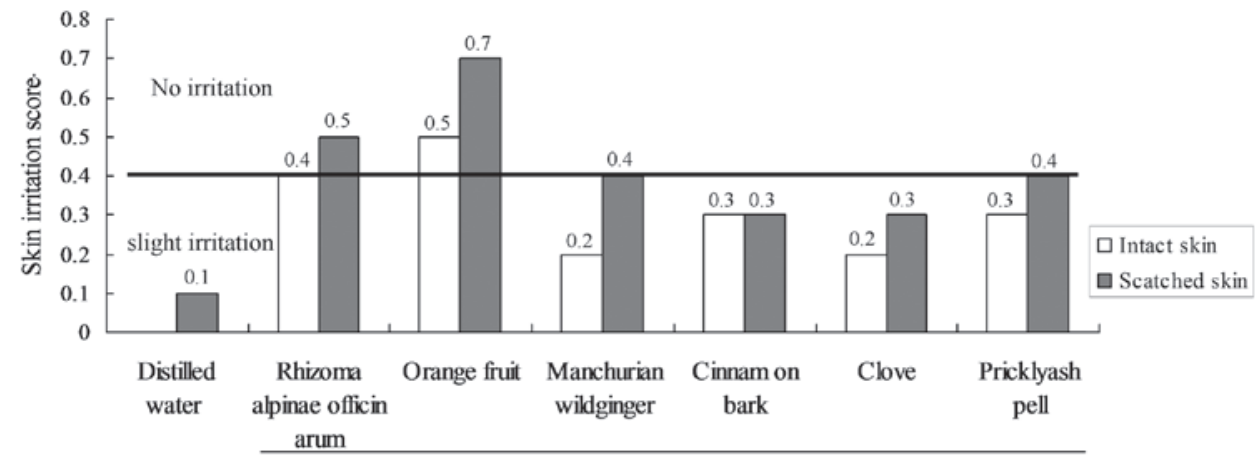

Volatile oil

Figure 3. Skin irritation scores of the intact skin and scratched skin treated with the various volatile oils. " $\mathrm{P}<0.05$, vs. distilled water. Black line indicates the division between no irritation (scores of 0-0.4) and slight irritation (scores of $>0.4$ ).
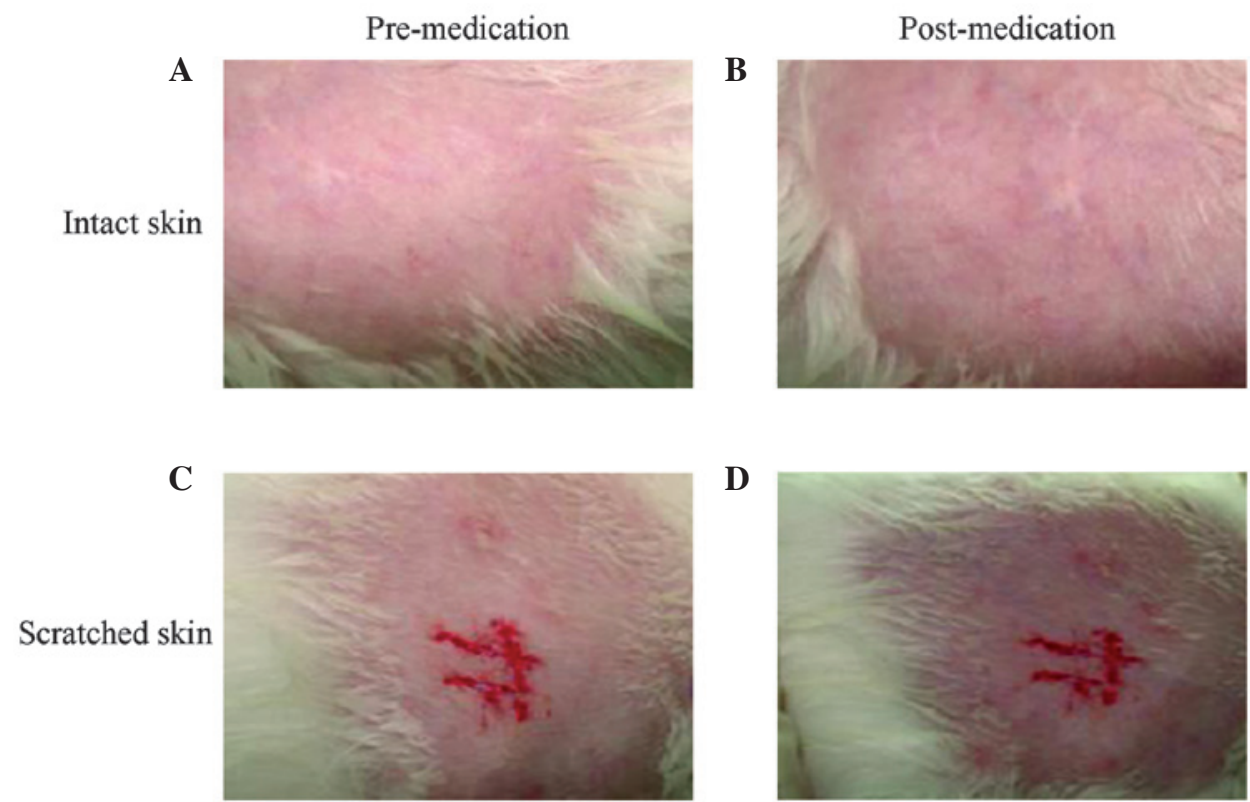

Figure 4. Effects of the clove volatile oil on the rabbit skin. (A) Intact skin prior to administration; (B) intact skin following administration; (C) scratched skin prior to administration; and (D) scratched skin following administration. 
volatile oils, the two species of Demodex were active, with the limbs maintaining an exercise status (Fig. 1). The average exercise frequency of each limb was $>15$ times/min. Following addition of the volatile oils, the polypides exercised intensively and contracted, deforming significantly (Fig. 1). The exercise frequency significantly increased to $>20 \mathrm{times} / \mathrm{min}$. The Demodex polypide was exercising in the volatile oils, experiencing a shortly excited state, which indicated an inhibitory state. Finally, the activity of Demodex decreased significantly, and a parasiticide outcome was achieved.

Lethal times of the volatile oils on Demodex. All six types of volatile oils were shown to kill the two species of Demodex within $30 \mathrm{~min}$. All the volatile oils demonstrated a significantly higher antiparasitic activity compared with the positive control of Xin Fumanling ointment (Fig. 2; $\mathrm{P}<0.05$ ). In addition, the antiparasitic activity of the volatile oil from Manchurian wild ginger and clove on Demodex folliculorum were significantly higher compared with that on Demodex brevis (Fig. 2; $\mathrm{P}<0.05$ ).

Volatile oils trigger no significant skin irritation. Results from the skin irritation test indicated that Rhizoma Alpiniae Officinarum and orange fruit induced only a slight irritation. Furthermore, Manchurian wild ginger, cinnamon bark, clove and pricklyash peel were not shown to induce any irritation for the intact skin or scratched skin, with scores of $<0.4$ points (Fig. 3). In particular, the clove volatile oil was not found to trigger any irritation, with effects equivalent to those of distilled water.

No significant adverse effects were observed with the volatile oil-treated skin. After one week of observation, no adverse effects were observed on the intact skin and scratched skin of the rabbits. The weight, hair, skin, respiratory system and limb activity of the rabbits were not shown to exhibit a significant abnormality (Fig. 4).

\section{Discussion}

A number of drugs have been applied as anti-Demodex therapy, including 1-2\% metronidazole (17), 2 or $5 \%$ permethrin (18), $200 \mathrm{~g} / \mathrm{kg}$ ivermectin (19), amongst others. However, the aforementioned drugs require complex synthetic technology, and have been shown to exert certain adverse effects following application. Chinese medicine shares the benefits of reduced side effects, minor toxicity and few side effects; thus, may play an important role in anti-Demodex therapy. To date, a number of Chinese drugs have been synthesized for Demodex therapy in China.

Volatile oil is a type of oily liquid with characteristics of extensive bioactivity and an aromatic odor (20). The present study selected six types of volatile oils, extracted using a distillation method, and observed their anti-Demodex activity. The results indicated that all the volatile oils possessed more intense anti-Demodex activity compared with the positive control. Among these volatile oils, the clove volatile oil provided the most intense anti-Demodex activity.

A previous study (21) also investigated the effects of clove, orange fruit, Manchurian wildginger, cinnamon bark,
Rhizome Alpiniae Officinarum and pricklyash peel. However, the anti-Demodex function has not yet been fully explored. In the present study, clove volatile oil was adopted as the therapeutic drug, which was shown to trigger significant anti-Demodex activity compared with the Xin Fumanling ointment. According to the criteria outlined by the National Health and Family Planning Commission of China (Beijing, China), all maquillage and drugs designed for the skin should not cause mucosal irritation and damage. In the present study, a skin irritation test was performed to identify the safety of the volatile oils. From the irritation test results, it was found that no erythema or edema were observed in the intact skin, and only slight erythema was observed in the scratched skin following the application of clove volatile oil. An acute toxicity test was applied to analyze the toxicity and adverse effects. The results revealed that the clove volatile oil did not exhibit acute toxicity on the rabbit skin. Clove resources are very rich, and the volatile oil can be extracted conveniently. The low toxicity and safety demonstrate the feasibility of using clove volatile oil in clinical and basic studies.

In conclusion, Chinese crude medicine volatile oil, particularly that for clove, demonstrated a marked anti-Demodex activity. The clove volatile oil was shown to kill Demodex effectively and safely in vivo.

\section{Acknowledgements}

The study was supported by a grant from the Science and Technology Program from the Department of Education, Heilongjiang Province (no. 12521628).

\section{References}

1. Baima B and Sticherling M: Demodicidosis revisited. Acta Derm Venereol 82: 3-6, 2002.

2. Kim JH, Chun YS and Kim JC: Clinical and immunological responses in ocular demodecosis. J Korean Med Sci 26: 1231-1237, 2011.

3. English FP and Nutting WB: Demodicosis of ophthalmic concern. Am J Ophthalmol 91: 362-372, 1981.

4. Liu J, Sheha H and Tseng SC: Pathogenic role of Demodex mites in blepharitis. Curr Opin Allergy Clin Immunol 10: 505-510, 2010.

5. Forton F, Germaux MA, Brasseur T, De Liever A, Laporte M, Mathys C, Sass U, Stene JJ, Thibaut S, Tytgat M and Seys B: Demodicosis and rosacea: epidemiology and significance in daily dermatologic practice. J Am Acad Dermatol 52: 74-87, 2005.

6. Yang G, Geng C, Li Y, Liu A and Chen W: Multiple myeloma with extramedullary plasmacytoma invading the skin and eyeballs following autologous stem cell transplantation: A case report. Exp Ther Med 6: 883-886, 2013.

7. Morrás PG, Santos SP, Imedio IL, Echeverría ML and Hermosa JM: Rosacea-like demodicidosis in an immunocompromised child. Pediatr Dermatol 20: 28-30, 2003.

8. Erbagci Z, Erbagci I and Erkiliç S: High incidence of demodicidosis in eyelid basal cell carcinomas. Int J Dermatol 42: 567-571, 2003.

9. Zhao GJ, Hong GL, Liu JQ, Lu Y and Lu ZQ: Septic shock due to community-acquired Pseudomonas aeruginosa necrotizing fasciitis: A case report and literature review. Exp Ther Med 7: 1545-1548, 2014

10. Gao YY, Di Pascuale MA, Li W, Baradaran-Rafii A, Elizondo A, Kuo CL, Raju VK and Tseng SC: In vitro and in vivo killing of ocular Demodex by tea tree oil. Br J Ophthalmol 89: 1468-1473, 2005.

11. Coelho JP, Cristino AF, Matos PG, Rauter AP, Nobre BP, Mendes RL, Barroso JG, Mainar A, Uriteta JS, Fareleira JM, Sovová $H$ and Palavra AF: Extraction of volatile oil from aromatic plants with supercritical carbon dioxide: experiments and modeling. Molecules 17: 10550-10573, 2012. 
12. Hu M, Zhang H, Feng B, Liu K and Guo S: Extraction of polysaccharides from Fomes officinalis Ames and their antitumor activity. Exp Ther Med 6: 451-454, 2013.

13. Gao YY, Di Pascuale M, Li W, et al: High prevalence of Demodex in eye lashes with cylindrical dandruff. Invest Ophthalmol Vis Sci 46: 3089-3094 2005.

14. Baranda L, González-Amaro R, Torres-Alvarez B, Alvarez C and Ramírez V: Correlation between $\mathrm{pH}$ and irritant effect of cleansers marketed for dry skin. Int J Dermatol 41: 494-499, 2002.

15. Yamamoto K, Igawa K, Sugimoto K, Yoshizawa $Y$, Yanagiguchi K, Ikeda T, Yamada S and Hayashi Y: Biological safety of fish (tilapia) collagen. Biomed Res Int 2014: 630757, 2014.

16. Lee SH: Evaluation of acute skin irritation and phototoxicity by aqueous and ethanol fractions of Angelica keiskei. Exp Ther Med 5: 45-50, 2013.
17. Koçak M, Yağli S, Vahapoğlu G and Ekşioğlu M: Permethrin 5\% cream versus metronidazole $0.75 \%$ gel for the treatment of papulopustular rosacea. A randomized double-blind placebo-controlled study. Dermatology 205: 265-270, 2002.

18. Forstinger C, Kittler H and Binder M: Treatment of rosacea-like demodicidosis with oral ivermectin and topical permethrin cream. J Am Acad Dermatol 41: 775-777, 1999.

19. Persi A and Rebora A: Metronidazole and Demodex folliculorum. Acta Derm Venereol 61: 182-183, 1981.

20. Liu X, Meng Q, Song H and Zhao T: A traditional Chinese herbal formula improves pressure ulcers in paraplegic patients: A randomized, parallel-group, retrospective trial. Exp Ther Med 5: 1693-1696, 2013

21. Liu JX, Li CP, Zhang H and Sun YH: Volatile oil of Chinese crude drug against human Demodex: effectiveness and safety. Di 2 Jun Yi Da Xue Xue Bao 29: 1048-1051, 2009 (In Chinese). 